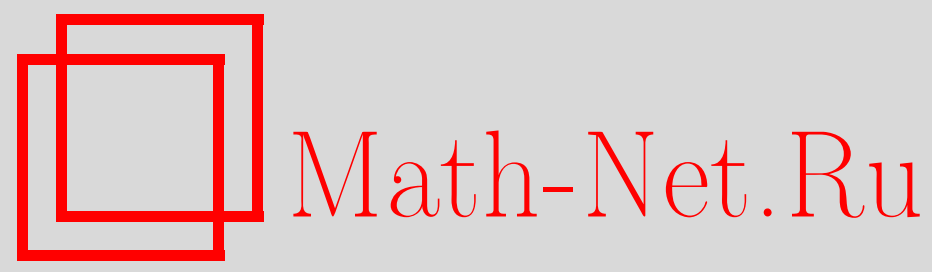

3. Д. Усманов, Связь многообразий решений общей и модельной обобщенных систем Коши-Римана с сингулярной точкой, Матем. заметки, 1999, том 66, выпуск 2, 302-307

DOI: https://doi.org/10.4213/mzm1168

Использование Общероссийского математического портала Math-Net.Ru подразумевает, что вы прочитали и согласны с пользовательским соглашением http://www.mathnet.ru/rus/agreement

Параметры загрузки:

IP : 3.85 .183 .62

26 апреля 2023 г., $07: 29: 29$

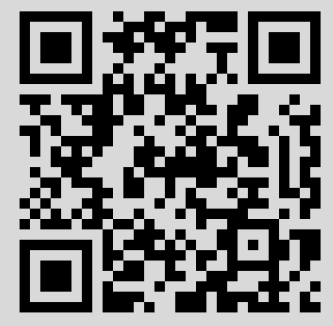




\title{
СВЯЗЬ МНОГООБРАЗИЙ РЕШЕНИЙ ОБЩЕЙ И МОДЕЛЬНОЙ ОБОБЩЕННЫХ СИСТЕМ КОШИ-РИМАНА С СИНГУЛЯРНОЙ ТОЧКОЙ
}

\section{3. Д. Усманов}

\begin{abstract}
Доказывается однозначная разрешимость вполне определенного двумерного интегрального уравнения. С помощью этого устанавливается взаимно однозначное соответствие между множествами непрерьвных решений систем вида $2 \bar{z} \partial_{\bar{z}} w-b(z) \bar{w}=0$ и $2 \bar{z} \partial_{\bar{z}} \Phi-b(0) \bar{\Phi}=0$, заданных в одной и той же области $G(z=0 \in G)$.

Библиограффия: 4 названия.
\end{abstract}

1. Введение. В статье рассматривается пара обобщенньх систем Коши-Римана следующего вида:

$$
\begin{aligned}
& 2 \bar{z} \partial_{\bar{z}} w-b(z) \bar{w}=0 \\
& 2 \bar{z} \partial_{\bar{z}} \Phi-b(0) \bar{\Phi}=0 .
\end{aligned}
$$

Обе системы определены в ограниченной области $G$ комплексного переменного $z$, содержащей внутри себя точку $z=0$. Предполагается, что $b(z) \in C(\bar{G}), b(0) \neq 0$ и $|b(z)-b(0)|<A|z|^{\alpha}, A, \alpha>0$, в некоторой малой окрестности $z=0$. Искомые функции $w(z)$ и $\Phi(z)$ рассматриваются из класса $D_{1, p}(G), p>2$, т.е. допускают обобщенные производные первого порядка по $z$ и $\bar{z}$, принадлежашие классу $L_{p}(\bar{G}), p>2$.

В статье установлен следующий результат.

Tеорема 1. Междду множествами $\{w(z)\}$ и $\{\Phi(z)\}$ решений из класса $D_{1, p}(G)$, $p>2$, систем (1) и (2) существует взаимно однозначное соответствие.

В [1] такое соответствие установлено лишь для частных случаев, которые характеризуются либо "малыми" размерами области $G$, либо значениями $b(z)$, "мало" отличаюшимися от 0 или $b(0)$. В таких ограничениях теория решений системы (1) развивается на базе соответствующей теории решений модельной системы (2) (см. [2]).

Доказательство существования упомянутого соответствия будет основываться на интегральном уравнении, полученном в [1]:

$$
w(z)=\Phi(z)+P_{G} \bar{w}, \quad z \in G,
$$

Работа выполнена при финансовой поддержке научного комитета HATO, грант OUTR.CRG № 960930 . 
в котором

$$
\begin{gathered}
P_{G} \bar{w}=S_{G}\left(\frac{b(\zeta)-b(0)}{2 \bar{\zeta}} \overline{w(\zeta)}\right), \quad \zeta=\xi+i \eta \\
S_{G} f=-\frac{1}{\pi} \iint_{G}\left(\frac{\Omega_{1}(z, \zeta)}{\zeta} f(\zeta)+\frac{\Omega_{2}(z, \zeta)}{\bar{\zeta}} \overline{f(\zeta)}\right) d \xi d \eta
\end{gathered}
$$

причем функции $\Omega_{1}$ и $\Omega_{2}$ задаются в виде

$$
\begin{aligned}
& \Omega_{1}=\left\{\begin{array}{l}
\frac{1}{2}\left(\frac{r}{\rho}\right)^{|\lambda|}+\sum_{k=1}^{\infty} \frac{P_{k} e^{i k(\varphi-\gamma)}+P_{-k} e^{-i k(\varphi-\gamma)}}{2 \mu_{k}}\left(\frac{r}{\rho}\right)^{\mu_{k}},|z|<|\zeta|, \\
-\frac{1}{2}\left(\frac{\rho}{r}\right)^{|\lambda|}-\sum_{k=1}^{\infty} \frac{P_{-k} e^{i k(\varphi-\gamma)}+P_{k} e^{-i k(\varphi-\gamma)}}{2 \mu_{k}}\left(\frac{\rho}{r}\right)^{\mu_{k}},|z|>|\zeta|,
\end{array}\right. \\
& \Omega_{2}=\left\{\begin{array}{l}
\frac{\lambda}{2|\lambda|}\left(\frac{r}{\rho}\right)^{|\lambda|}+\sum_{k=1}^{\infty} \frac{\lambda}{\mu_{k}}\left(\frac{r}{\rho}\right)^{\mu_{k}} \cos k(\varphi-\gamma),|z|<|\zeta|, \\
\frac{\lambda}{2|\lambda|}\left(\frac{\rho}{r}\right)^{|\lambda|}+\sum_{k=1}^{\infty} \frac{\lambda}{\mu_{k}}\left(\frac{\rho}{r}\right)^{\mu_{k}} \cos k(\varphi-\gamma),|z|>|\zeta|,
\end{array}\right.
\end{aligned}
$$

где $z=r e^{i \varphi}, \zeta=\rho e^{i \gamma}, \lambda=b(0), \mu_{k}^{2}=|\lambda|^{2}+k^{2}, P_{k}=\mu_{k}+k, P_{-k}=\mu_{k}-k$.

Соотношение (3), устанавливаюшее связь множеств $\{w(z)\}$ и $\{\Phi(z)\}$ решений из класса $D_{1, p}(G), p>2$, систем (1) и (2), сопоставляет каждому $w(z)$ единственное $\Phi(z)$. Что касается обратного отображения множества $\{\Phi(z)\}$ на $\{w(z)\}$, его свойства зависят от характера разрешимости уравнения (3) относительно $w(z)$. В [1] установлено, что оператор $P_{G}$ вполне непрерывен в пространстве $C(\bar{G})$ и отображает это пространство в себя. С учетом этого доказательство теоремы 1 сводится, очевидно, к проверке следующего утверждения.

ТЕОРема 2. Однородное интегральное уравнение

$$
w^{+}(z)=P_{G} \bar{w}^{+}, \quad z \in G,
$$

в классе непрерывных функций имеет только нулевое решение.

\section{2. Вспомогательные результаты.}

Лемма 1. Пусть $f(z) \in L_{p}(\bar{G}), p>2$. Тогда функиия $h(z)=S_{G} f$ непрерывна на плоскости $E$ и $h(z)=O\left(|z|^{-|\lambda|}\right)$ nрu $z \rightarrow \infty$.

В области $G$ функиия $h(z)$ принадлежит классу $D_{1, p}(G), p>2$, и является решением уравнения

$$
2 \bar{z} \partial_{\bar{z}} h-\lambda \bar{h}=2 \bar{z} f(z),
$$

а в области $E \backslash \bar{G}$ функиия $h(z)$ непрерывно дифферениируема и удовлетворяет уравнению

$$
2 \bar{z} \partial_{\bar{z}} h-\lambda \bar{h}=0
$$


ДокАЗАТЕЛЬСТво. Перечисленные свойства функции $h(z)$ заимствованы из $[1$, леммы 2,4$]$, и лишь одно из них - ее ассимптотика при $z \rightarrow \infty$ - нуждается в проверке.

Пусть $D_{0}=\left\{\zeta:|\zeta| \leqslant R_{0}\right\} \supset G$, где $R_{0}$ - максимальное расстояние от $\zeta=0$ до гранищы области $G$. Положим $z \in E \backslash \bar{D}_{0}$. Поскольку $|\zeta|<|z|$, имеем

$$
\Omega_{1}=-\left(\frac{\rho}{r}\right)^{|\lambda|} \Omega_{1}^{0}(z, \zeta), \quad \Omega_{2}=\left(\frac{\rho}{r}\right)^{|\lambda|} \Omega_{2}^{0}(z, \zeta)
$$

где

$$
\begin{aligned}
& \Omega_{1}^{0}=\frac{1}{2}+\sum_{k=1}^{\infty} \frac{P_{-k} e^{i k(\varphi-\gamma)}+P_{k} e^{-i k(\varphi-\gamma)}}{2 \mu_{k}}\left(\frac{\rho}{r}\right)^{\mu_{k}-|\lambda|} \\
& \Omega_{2}^{0}=\frac{\lambda}{2|\lambda|}+\sum_{k=1}^{\infty} \frac{\lambda}{\mu_{k}} \cos k(\varphi-\gamma)\left(\frac{\rho}{r}\right)^{\mu_{k}-|\lambda|}
\end{aligned}
$$

Очевидно, что $\Omega_{1}^{0}$ и $\Omega_{2}^{0}$ являются непрерьвными функциями по $z$ и $\zeta\left(z \in E \backslash \bar{D}_{0}\right.$ и $\left.\zeta \in G\right)$.

Осталось записать $h(z)$ в виде $h(z)=r^{-|\lambda|} h_{0}(z)$, где

$$
h_{0}(z)=\frac{1}{\pi} \iint_{G} \rho^{|\lambda|-1}\left(\Omega_{1}^{0}(z, \zeta) e^{-i \gamma} f(\zeta)-\Omega_{2}^{0}(z, \zeta) e^{i \gamma} \overline{f(\zeta)}\right) d \xi d \eta
$$

с тем, чтобы убедиться в справедливости требуемой асимптотики, поскольку из непрерьвности $\Omega_{1}^{0}$ и $\Omega_{2}^{0}$ следует непрерьвность функции $h_{0}(z)$ по $z \in E \backslash \bar{D}_{0}$, в частности при $z=\infty$. Однако, в дальнейшем нам понадобится оценка для $h(z)$.

Применяя неравенство Гёльдера, получим

$$
\begin{aligned}
\left|h_{0}(z)\right| \leqslant & \frac{1}{\pi}\left(\iint_{G} \rho^{(|\lambda|-1) r} d \xi d \eta\right)^{1 / r}\left(\iint_{G}|f(\zeta)|^{p} d \xi d \eta\right)^{1 / p} \\
& \left.\times\left(\iiint_{G}\left|\Omega_{1}^{0}(z, \zeta)\right|^{q} d \xi d \eta\right)^{1 / q}+\left(\iint_{G}\left|\Omega_{2}^{0}(z, \zeta)\right|^{q} d \xi d \eta\right)^{1 / q}\right),
\end{aligned}
$$

где $1 / p+1 / q+1 / r=1, p, q>2$ и $1<r<2$. Оценивая в правой части этого неравенства первый сомножитель, а также третий сомножитель с помощью неравенств Гёльдера и Минковского, для функции $h(z)$ получаем

$$
|h(z)|<M L_{p}(f, G)|z|^{-|\lambda|}, \quad z \in E \backslash \bar{D}_{0},
$$

где $M=M\left(|\lambda|, R_{0}\right)$ - константа, зависящая от $|\lambda|$ и $R_{0}$, а $L_{p}(f, G)$ - норма $f(z)$ в пространстве $L_{p}(\bar{G})$.

Воспользуемся леммой 1 для описания свойств решений однородного интегрального уравнения (4). Принимая во внимание связь операторов $P_{G}$ и $S_{G}$, устанавливаем, что всякое непрерьвное решение $w^{+}(z)$ уравнения (4) принадлежит классу $D_{1, p}(G), p>2$, и удовлетворяет согласно (5) уравнению (1). В таком случае из теоремы 1.1 [2, c. 74] следует, что $w^{+}(z)$ должно подчиняться априорной асимптотике

$$
w^{+}(z)=O\left(|z|^{|\lambda|}\right), \quad z \rightarrow 0 \text {. }
$$


Из леммы 1 вьводится также, что $w^{+}(z)$ непрерьвно продолжима в области $E \backslash \bar{G}$ вполне определенной непрерывной функцией $w^{-}(z)$, удовлетворяющей уравнению (6). В соответствии с теорией эллиптических систем $w^{-}(z)$ является аналитической функцией по $z$ и $\bar{z}$ в $E \backslash \bar{G}$. Еще одно важное свойство функции $w^{-}(z)$ следует из неравенства (7). Записывая его для решений уравнения (4), положим

$$
f(z)=(b(z)-b(0)) \frac{\overline{w^{+}(z)}}{2 \bar{z}}, \quad h(z) \equiv w^{-}(z) .
$$

Тогда

$$
\left|w^{-}(z)\right|<M L_{p}\left(\frac{b(z)-b(0)}{2 \bar{z}}, G\right)\left\|w^{+}\right\|_{C(G)}|z|^{-|\lambda|} .
$$

Таким образом, на плоскости $E$ задается функция

$$
W(z)= \begin{cases}w^{+}(z), & z \in \bar{G} \\ w^{-}(z), & z \in E \backslash \bar{G},\end{cases}
$$

являющаяся решением системы

$$
2 \bar{z} \partial_{\bar{z}} W-B(z) \bar{W}=0
$$

в которой

$$
B(z)=\left\{\begin{array}{l}
b(z), \quad z \in \bar{G}, \\
b(0), \quad z \in E \backslash \bar{G} .
\end{array}\right.
$$

ЛЕмма 2. Если решение системы (10) непрерывно на всей комлексной плоскости и подчиняется условиям (8) $u(9)$, то $W(z) \equiv 0$.

Из леммы 2 следует, что $w^{+}(z) \equiv 0, z \in G$, а значит, справедлива теорема 2.

3. ДоКАЗАТЕЛЬСТво ЛЕммЫ 2. Предположим противное, т.е. существует хотя бы одно решение $W_{0}(z) \not \equiv 0, z \in E$. Пусть $D=\{z:|z| \leqslant R\}-$ круг, радиус $R$ которого превосходит максимальное расстояние $R_{0}$ от точки $z=0$ до границы области $G$, т.е. $R>R_{0}$. Представим функцию $W_{0}(z), z \in D$, в виде [3]

$$
W_{0}(z)=\Psi(z) e^{\omega(z)},
$$

где $\Psi(z)$ - аналитическая функция комплексного переменного $z$,

$$
\begin{gathered}
\omega(z)=-\frac{1}{2 \pi} \iint_{D} \frac{f(\zeta) d \xi d \eta}{\bar{\zeta}(\zeta-z)}, \quad \zeta=\xi+i \eta, \\
f(z)=\left\{\begin{array}{l}
B(z) \frac{\bar{W}_{0}(z)}{W_{0}(z)} \\
B(z)
\end{array} \text { для точек } z, \text { в которых } W_{0}(z) \neq 0,\right.
\end{gathered}
$$

Из (11) заключаем, что $\Psi(z)$ является непрерывной функцией вне точки $z=0$, поскольку таковой является $\omega(z)$. Для того чтобы описать поведение $\Psi(z)$ при $z=0$ и $|z|=R$, воспользуемся неравенством

$$
\iint_{D} \frac{d \xi d \eta}{|\zeta| \cdot|\zeta-z|}<2 \pi \ln \frac{R}{|z|}+5 \pi \ln 2+\pi \ln (1+\sqrt{2})
$$


проверка которого производится далее в п. 4 .

С учетом специфики коэффициента $B(z)$ системы $(10)$ получаем

$$
|\omega(z)|<\frac{|b(0)|}{2 \pi} \iint_{D} \frac{d \xi d \eta}{|\zeta| \cdot|\zeta-z|}+\frac{1}{2 \pi} \iint_{G} \frac{|b(\zeta)-b(0)|}{|\zeta| \cdot|\zeta-z|} d \xi d \eta .
$$

Оценивая первый интеграл с помощью (12) и принимая во внимание ограниченность второго интеграла, вьводим

$$
|\omega(z)|<|\lambda| \ln \frac{R}{|z|}+N
$$

Здесь, как и ранее, $\lambda=b(0)$ и $N$ - константа, не зависящая от $R$.

Теперь из (11) находим

$$
|\Psi(z)|<\left(\frac{R}{|z|}\right)^{|\lambda|} e^{N}\left|W_{0}(z)\right|, \quad z \in D .
$$

Используя (8), устанавливаем, что $\Psi(z)$ ограничена в точке $z=0$. Если распространить это неравенство на точки $z \in \partial D=\{z:|z|=R\}$ и воспользоваться $(9)$, получим

$$
|\Psi(z)|<M e^{N}\left\|w^{+}\right\|_{C(G)} L_{p}\left(\frac{b(\zeta)-b(0)}{2 \bar{\zeta}}, G\right) R^{-|\lambda|} .
$$

В силу принципа максимума модуля для аналитических функций последнее неравенство имеет место для всех точек $z \in D$.

Пусть $\varepsilon$ - сколь угодно малое положительное число. За счет выбора достаточно большого $R=R(\varepsilon)$, очевидно, можно добиться вьполнения условия $|\Psi(z)|<\varepsilon$ для $z \in D$, а значит, и для $z \in G$. Тогда $\Psi(z) \equiv 0$ по крайней мере для $z \in G$. Тем самым, из (11) следует, что $W_{0}(z)=w^{+}(z) \equiv 0$ для $z \in G$. Но тогда согласно теории эллиптических систем $W_{0}(z) \equiv 0$ в любой окрестности точки $z=0$. Лемма 2 , а вместе с ней теоремы 2 и 1 доказаны.

4. ДоКАЗАТЕЛЬСТво НЕРАВЕНСТВА (12). Обозначим через $J$ интеграл в левой части неравенства (12). Осуществляя переход к полярным координатам $z=r e^{i \varphi}, \zeta=\rho e^{i \gamma}$, представим $J$ в виде

$$
J=\int_{-\pi / 2}^{\pi / 2} J_{1} d \alpha+\int_{\pi / 2}^{3 \pi / 2} J_{2} d \alpha
$$

где

$$
J_{k}=\int_{0}^{R} g(\rho, r, \alpha) d \rho, \quad k=1,2,
$$

причем $\alpha=\gamma-\varphi, g(\rho, r, \alpha)=\left((\rho-r \cos \alpha)^{2}+r^{2} \sin ^{2} \alpha\right)^{-1 / 2}$.

Оценивая $J_{1}$, необходимо иметь в виду, что при $\zeta=z(\rho=r, \alpha=0)$ подьнтегральное выражение обращается в бесконечность. Поэтому

$$
\begin{aligned}
J_{1} & =\lim _{\varepsilon_{1} \rightarrow 0} \int_{0}^{r \cos \alpha-\varepsilon_{1}} g(\rho, r, \alpha) d \rho+\lim _{\varepsilon_{2} \rightarrow 0} \int_{r \cos \alpha+\varepsilon_{2}}^{R} g(\rho, r, \alpha) d \rho \\
& =-2 \ln (r|\sin \alpha|)+\ln (r(1+\cos \alpha))+\ln \left(R-r \cos \alpha+g^{-1}(R, r, \alpha)\right) \\
& \leqslant \ln \frac{R}{r}+\ln 2-2 \ln |\sin \alpha|+\ln \left(1-\frac{r}{R} \cos \alpha+g^{-1}\left(1, \frac{r}{R}, \alpha\right)\right) .
\end{aligned}
$$


Оценим $J_{2}$, учитывая условие $\cos \alpha \leqslant 0(\pi / 2 \leqslant \alpha \leqslant 3 \pi / 2)$ :

$$
J_{2} \leqslant \int_{0}^{R} \frac{d \rho}{\rho+r|\cos \alpha|}=\ln |R+r| \cos \alpha||-\ln (r|\cos \alpha|) \leqslant \ln \frac{R}{r}+\ln 2-\ln |\cos \alpha| .
$$

Применяя в (13) полученные оценки для $J_{1}$ и $J_{2}$, а также соотношения

$$
\begin{gathered}
\int_{0}^{\pi / 2} \ln \cos \alpha d \alpha=\int_{0}^{\pi / 2} \ln \sin \alpha d \alpha=-\frac{\pi}{2} \ln 2 \\
\int_{-\pi / 2}^{\pi / 2} \ln \left(1-\frac{r}{R} \cos \alpha+g^{-1}\left(1, \frac{r}{R}, \alpha\right)\right) d \alpha \leqslant \ln (1+\sqrt{2})
\end{gathered}
$$

приходим к неравенству (12).

Отметим, что оценка (12) более точна по сравнению с оценками, имеющимися в [3, с. 55] и $[4$, с. 104].

\section{СПИСОК ЦИТИРОВАННОЙ ЛИТЕРАТУРЫ}

[1] Усманов З. Д. Об одном классе обобщенных систем Коши-Римана с сингулярной точкой // Сиб. матем. ж. 1973. Т. 14. № 5. С. 1076-1087.

[2] Усманов З. Д. Обобщенные системы Коши-Римана с сингулярной точкой. Душанбе: ТаджикНИИНТИ, 1993.

[3] Векуа И.Н. Обобщенные аналитические функции. М.: Физматгиз, 1959.

[4] Михайлов Л. Г. Новый класс особых интегральных уравнений и его применения к дифференциальным уравнениям с сингулярньми коэффициентами. Душанбе: Изд. АН ТаджССР, 1963.

Институт математики АН республики Таджикистан, г. Душанбе

Поступило

E-mail: usmanov@td.silk.org

01.04 .97

Исправленный вариант

19.10 .98 